\title{
Populismus auf Facebook
}

\section{Ein explorativer Vergleich der Parteien- und Anschlusskommunikation von AfD, CDU und SPD}

\author{
Elina Spieß • Dennis Frieß • Anne Schulz
}

Online publiziert: 13 . Juli 2020

(C) Der/die Autor(en) 2020

Zusammenfassung Die Beziehung zwischen Medien und Populismus ist in den letzten Jahren vermehrt in den Fokus wissenschaftlichen Interesses gerückt. Mit Blick auf das Internet und soziale Medien wurde schon früh eine strukturelle Kompatibilität für populistische Kommunikation theoretisiert. Entsprechend haben Studien auch empirisch untersucht, in welchem Ausmaß politische Akteure populistische Inhalte online publizieren. Der explorative Beitrag knüpft an diese Forschungslinie an und nimmt die offiziellen Facebook-Seiten der AfD, CDU und SPD in den Blick. Dabei wird der Populismusgehalt a) der dort in Posts platzierten Parteien-Kommunikation selbst sowie b) der Anschlusskommunikation, die in Nutzerkommentaren unter entsprechenden Posts sichtbar wird, vergleichend analysiert. Schließlich exploriert der Beitrag c) inwiefern Populismus in der Parteienkommunikation den Populismusgehalt der Anschlusskommunikation beeinflusst. Die Ergebnisse einer Inhaltsanalyse von 224 Parteienposts und 448 Nutzerkommentaren lassen erkennen, dass die AfD (im Vergleich zu CDU und SPD) am häufigsten und intensivsten populistische Inhalte über Facebook verbreitet. Nutzerkommentare sind jedoch insbesondere auf der CDU Facebook-Seite populistisch aufgeladen. Ein positiver Zusammenhang zwischen dem Populismusgehalt der Initialposts und dem Populismusgehalt der Nutzerkommentare kann für die AfD und die CDU festgestellt werden, jedoch nicht bei der SPD. Zudem offenbart die Analyse, dass auf den Sei-

\footnotetext{
E. Spieß, M.A.

Institut für Kommunikationswissenschaft, Westfälische Wilhelms-Universität Münster, Münster, Deutschland

E-Mail: Elina-spiess@web.de

D. Frieß, M.A. $(\bowtie)$

Institut für Sozialwissenschaften, Heinrich-Heine-Universität Düsseldorf, Düsseldorf, Deutschland E-Mail: Dennis.friess@hhu.de

Dr. A. Schulz

Reuters Institut für Journalismusforschung, Universität Oxford, Oxford, Großbritannien

E-Mail: anne.schulz@politics.ox.ac.uk
} 
ten der CDU und SPD viel häufiger kritische Oppositionsstimmen zu finden sind als auf der AfD-Facebookseite. Diese oppositionellen Kommentare sind wiederum signifikant häufiger populistisch.

\section{Populism at Facebook}

An exploratory comparison of the party- and user communication of AfD, CDU, and SPD

Abstract The relationship between media and populism has become a popular research topic in recent years. With regard to the Internet and social media, a structural compatibility for populist communication was theorized early on. Accordingly, studies have empirically examined the extent to which political actors publish populist content online. This exploratory study follows this line of research by investigating Facebook pages of the German parties AfD, CDU and SPD. In detail we focus on the populist communication markers within a) the Facebook postings by the three parties and b) in the subsequent user comments. In addition to that the study explores c) whether populism in party postings stimulates populistic communication in the subsequent user discussions. The results of a content analysis of 224 party posts and 448 user comments show that the AfD (compared to the CDU and SPD) distributes populist content both more often and intensively via Facebook. However, populistic user comments are particularly found on the CDU Facebook page. A positive correlation between the populism in party postings and the populism in user comments can be found for the AfD and the CDU, but not for the SPD. Additionally, the analysis reveals that oppositional voices are much more likely to be found on the CDU and SPD pages than on the AfD Facebook page. These oppositional comments contain significantly more populistic markers.

\section{Einleitung}

Europa erlebt ein Erstarken populistischer Kräfte. Nebst den mit der fortschreitenden Globalisierung einhergehenden gesellschaftlichen, sozialen und ökonomischen Veränderungen, werden auch der Einfluss digitaler Medien sowie der Wandel der Medienlandschaft insgesamt als Faktoren für den Erfolg populistischer Parteien und Akteure genannt (Mazzoleni 2008; Aalberg et al. 2017). Die Beziehung zwischen Medien und Populismus ist in den letzten Jahren vermehrt in den Fokus wissenschaftlichen Interesses gerückt (Krämer 2018). So widmeten sich einige Studien der Untersuchung von Nachrichtenmedien als Plattform für politischen Populismus (Bos et al. 2010; Esser et al. 2016; Wettstein et al. 2018a). Andere Studien untersuchten, ob und inwiefern Nachrichtenmedien populistische Kommunikationsstrategien adaptieren und so selbst zu populistischen Akteuren werden (Bos und Brants 2014; Hameleers et al. 2017a; Wettstein et al. 2018b). Auch die Wirkung von populistischer Kommunikation auf die öffentliche Meinungsbildung ist jüngst Gegenstand empirischer Forschung geworden (Müller et al. 2017; Wirz et al. 2018; Wirz 2018). Schließlich haben Arbeiten mit Blick auf die Online-Welt untersucht, in welchem Ausmaß politische Akteure populistische Inhalte online publizieren (Engesser et al. 
2017; Ernst et al. 2017). Der vorliegende Beitrag knüpft an diese zuletzt genannte Forschungslinie an und richtet den Blick auf die Facebook-Kommunikation von Parteien in Deutschland.

Parteien gelten im politischen System der Bundesrepublik als zentrale Akteure. Obgleich Parteien von Verfassungswegen lediglich eine Mitwirkung an der politischen Willensbildung zugestanden wird (Art. 21 Abs. 1. GG), spielen sie de facto in fast allen Bereichen des politischen Alltags eine hervorgehobene Rolle, weshalb Deutschland als Parteiendemokratie bezeichnet wird (Alemann et al. 2018). Als Intermediäre haben Parteien eine wichtige Funktion bei der Interessensaggregation und übernehmen eine Scharnierfunktion zwischen Gesellschaft und Politik (Sartori 1976). Vor dem Hintergrund sich verändernder Legitimations- und Repräsentationsansprüche (Sarcinelli 2013) stehen Parteien vor der Herausforderung, einen responsiveren Kommunikations- und Entscheidungsstil zu pflegen. Dabei spielen die interaktiven Potenziale des Internets und insbesondere soziale Medien eine immer wichtigere Rolle (Chadwick und Stromer-Galley 2016; Emmer 2019; Staender et al. 2019; Stier et al. 2018).

Soziale Medien gehören, auch abseits des Wahlkampfes, mittlerweile zum festen Kommunikationsrepertoire von Parteien. Mit sozialen Medien stehen ihnen Kanäle zur öffentlichen Kommunikation zur Verfügung, über die sie direkt und ungefiltert Botschaften senden, über die eigene Arbeit informieren und Diskussionen auslösen können. Durch ihre besondere Stellung im politischen System kommt Parteien bei der Initiierung öffentlicher Diskussionen eine besondere Verantwortung zu.

Ihre Präsenz auf den verschiedenen politischen Ebenen, ihre stark kommunikative Orientierung sowie ihre enge Verbundenheit sowohl mit der Lebenswelt der Bürgerinnen und Bürger als auch mit dem politischen Entscheidungszentrum macht sie zu Schlüsselorganisationen in der politischen Kommunikation und für politische Entscheidungen. (Donges 2008, S. 27-28)

Parteien sind in diesem Kontext nicht nur als Interessensaggregatoren zu verstehen, sondern auch als Diskursstifter, wenn sie gesellschaftlich relevante Themen auf die Agenda heben. Über soziale Medien werden diese Diskurse unmittelbar beobachtbar und - auf Grund der interaktiven und offenen Struktur sozialer Medien - potenziell dynamischer und konflikthafter (Neuberger 2014). Da Parteien stets im Fokus medialer Beobachtung stehen, können parteiinitiierte Diskussionen große Reichweiten erlangen und entsprechend die politische (Diskussions-)Kultur beeinflussen. Darum ist es wichtig, danach zu fragen, welche Rolle populistische Inhalte auf Parteiseiten spielen. In diesen Zusammenhang hat auch Waisbord (2018) darauf hingewiesen, dass Populismus eine Reihe von Problemen für demokratische Kommunikation mit sich bringt. Die in populistischer Kommunikation angelegte Agonistik, Volkshomogenität sowie die Simplifizierung komplexer Sachverhalte auf einfache Lösungen unterwandere Toleranz, Meinungs- und Pressefreiheit sowie Pluralität und stelle dementsprechend eine Gefahr für liberale Demokratien dar (Waisbord 2018, S. 30). Vor diesem Hintergrund ist es auch aus einer politisch-kulturellen Perspektive relevant zu fragen, ob Parteien populistische Elemente in ihrer Online-Kommunikation nutzen und wie sich dies auf die Anschlusskommunikation in sozialen Medien auswirkt. 
Zwischen sozialen Medien und Populismus wurde schon früh eine strukturelle Kompatibilität theoretisiert (Bimber 1998), die vor dem Hintergrund aktuellerer politischer Geschehnisse weiter ausgearbeitet wurde (Gerbaudo 2018). Zum einen können populistische Akteure mit ihren Inhalten diverse Adressaten ungefiltert erreichen, wobei sie den Journalismus als Gatekeeper umgehen. Zum anderen offerieren Aufbau und Funktionslogik sozialer Medien eine Reihe von Feedbackmöglichkeiten für Nutzer und suggerieren so eine Nähe zwischen Kommunikator und Adressat (Engesser et al. 2017; Ernst et al. 2017; Waisbord und Amado 2017). Daraus folgert Bartlett (2014, S. 106): ,Social media is in many ways the ideal medium for populist parties“.

Vor dem Hintergrund dieser theoretisierten Kompatibilität haben Studien in den vergangenen Jahren soziale Medien als Gelegenheitsstrukturen für populistische Kommunikation untersucht (Blassnig et al. 2018; Ernst et al. 2019a; Schmuck und Hameleers 2019). Erfasst wurde im Wesentlichen die Prävalenz populistischer Kommunikation durch politische Akteure auf Plattformen wie Facebook und/oder Twitter und dies oft im Vergleich zu deren Vorkommen in anderen Medien, wie beispielsweise Talkshows oder Zeitungen (Blassnig et al. 2018; Ernst et al. 2019b; van Kessel und Castelein 2016). Die Studien zeigen, dass populistische Kommunikation insbesondere auf Facebook reüssiert und dass sie dort hauptsächlich von populistischen Parteien oder deren Mitgliedern verbreitet wird. Weitaus weniger Studien haben sich bisher mit den Reaktionen der Nutzer auf populistische Kommunikation in sozialen Medien auseinandergesetzt. Mit einem verwandten Forschungsanliegen erfassten Blassnig et al. (2019a) den Populismusgehalt von Leserkommentaren unter Online-Nachrichtenartikeln. Die Studie konnte zeigen, dass diese Leserkommentare oft populistischer aufgeladen waren als der Artikel, auf den sie sich bezogen. Eine weitere Untersuchung stellte fest, dass populistische Inhalte in Nachrichtenartikeln nicht nur die Anzahl der Kommentare steigern, sondern auch zu mehr populistischen Nutzerkommentaren führen (Blassnig et al. 2019b). Die Ergebnisse deuten darauf hin, dass Populismus sowohl Aufmerksamkeit steigern kann, als auch einen Ansteckungseffekt auf die Anschlusskommunikation in Nutzerkommentaren hat. Allerdings gelten diese Befunde für Populismus im Kontext von Nachrichtenartikeln und den entsprechenden Kommentarspalten. Der Populismusgehalt der Anschlusskommunikation, die sich in den Nutzerkommentaren unter den Posts politischer Parteien wiederfinden, ist bisher nicht untersucht worden. Ebenso wenig wie die Frage, ob Parteienposts einen Einfluss auf den Ton der Anschlusskommunikation nehmen.

Die vorliegende Studie setzt an diesem Forschungsdesiderat an, indem sie neben der originären Partei-Kommunikation auch die Anschlusskommunikation in Nutzerkommentaren auf Facebook untersucht. Die explorativ angelegte Studie nimmt dabei die offiziellen Facebook-Seiten der AfD, CDU und SPD in den Blick und vergleicht den Populismusgehalt a) der dort in Posts platzierten Parteien-Kommunikation selbst sowie b) der Anschlusskommunikation in Nutzerkommentaren. Schließlich exploriert der Beitrag c), inwiefern die populistische Parteienkommunikation den Po- 
pulismusgehalt in der Anschlusskommunikation beeinflusst. ${ }^{1}$ Die Studie stellt die folgenden Forschungsfragen:

1. Wie unterscheiden sich die Posts der AfD, CDU und SPD sowie die Nutzerkommentare auf den jeweiligen Facebook-Seiten in Hinblick auf ihren populistischen Gehalt?

2. Beeinflusst der Populismusgehalt in den Parteienposts den Populismusgehalt in der in Nutzerkommentaren festgehaltenen Anschlusskommunikation?

Die erste Forschungsfrage soll ermitteln, inwieweit Parteien in sozialen Medien auf populistische Kommunikationsinhalte zurückgreifen. Dabei gilt es nicht nur zu betrachten, ob als populistisch deklarierte Parteien, wie beispielsweise die AfD (Bebnowski 2015; Berbuir et al. 2015), tatsächlich populistisch über soziale Medien kommunizieren, sondern auch, ob etablierte Parteien, wie CDU und SPD, die für gewöhnlich nicht als populistische Parteien behandelt werden, populistische Elemente in ihre Online-Kommunikation einfließen lassen. Mit diesem Vorgehen folgen wir einer Vorlage von Ernst et al. (2017), die zeigen konnten, dass die Facebook- und Twitter-Kommunikation populistischer Partien am rechten und linken Rand tatsächlich populistischer aufgeladen ist als die Kommunikation von nicht-populistischen Oppositions- und Regierungspartien. Wir haben hier die Möglichkeit diese Beobachtungen aus dem Jahr 2015 mit Daten aus den Jahren 2016/2017 noch einmal nachzuvollziehen.

Darüber hinaus soll auch der Populismusgehalt der Nutzerkommentare auf entsprechenden Facebook-Seiten untersucht werden. Die zweite Forschungsfrage der Untersuchung widmet sich dabei den möglichen Konsequenzen populistischer Kommunikation für die öffentliche Anschlusskommunikation in Sozialen Medien. Eine solche Untersuchung ist bisher nicht unternommen worden. Es gilt zu beantworten, ob und inwiefern populistische Posts auf Facebook populistische Nutzerkommentare nach sich ziehen. Da Kommentare eine Orientierungsfunktion für andere Nutzer haben können, erscheint deren Untersuchung besonders relevant. So wäre beispielsweise denkbar, dass sich die Leser solcher Kommentare in ihrer Meinungsbildung und Meinungsäußerung beeinflussen lassen (Noelle-Neumann 1980; Schulz und Rössler 2012) oder die Kommentare nutzen, um die eigene Position zu verifizieren (Springer et al. 2015). Ferner haben Untersuchungen im Feld der Nutzerkommentarforschung gezeigt, dass die (oft geringe) Qualität von Nutzerkommentaren eine Reihe von negativen Wirkungen auf deren Leser haben kann (Hsueh et al. 2015; Ziegele et al. 2018).

Vor dem Hintergrund der skizzierten Erkenntnisse aus angrenzenden Forschungsfeldern scheint es daher relevant, Nutzerkommentare auch im Kontext populistischer Kommunikation zu analysieren. Die Frage, ob populistische Parteienkommentare auch die daran anschließende Debatte populistisch auflädt, ist auch aus einer demokratietheoretischen Perspektive von Interesse. Auch wenn Populismus als legitime Facette von Demokratie verstanden werden kann (Canovan 1999; Mény und Surel

\footnotetext{
1 Die explorativ angelegte Untersuchung nimmt lediglich drei Parteien in den Blick. Dabei ist kritisch anzumerken, dass eine Oppositionspartei (AfD) mit zwei Regierungsparteien (CDU \& SPD) verglichen wird.
} 
2002), beschreibt die überwiegende Mehrheit Populismus als eine Gefahr, die zentrale Aspekte der liberalen Demokratie (Meinungs- und Pressefreiheit, Pluralismus, Toleranz, Gewaltenteilung, Wahrhaftigkeit) unterwandern und politische Kommunikation negativ beeinflussen kann (Abts und Rummens 2007; Waisbord 2018).

\section{Theoretische Annäherung an Populismus}

Um im Folgenden die Prävalenz populistischer Kommunikation in der FacebookKommunikation von Parteien und Nutzern empirisch erfassen zu können, muss zunächst eine theoretische Klärung des Populismus-Begriff erfolgen. Die Problematik, Populismus zu definieren, begleitet die Populismusforschung seit Anbeginn. Gründe dafür mögen in der hohen Kontextabhängigkeit des Phänomens liegen (Taggart 2004). Dies wird etwa auch in der historischen Rückschau deutlich, die zeigt, dass Populismus in jeweils sehr unterschiedlichen Kulturen und Formen auftreten kann (Canovan 1981; Taggart 2004). In diesem Abschnitt soll nun vorerst eine theoretische Definition des Begriffs geleistet werden, um daran anschließend verschiedene Dimensionen eines empirischen Populismusbegriffs herauszuarbeiten.

Seiner ihm innewohnenden Ambiguität geschuldet, ist Populismus in der Literatur theoretisch auf sehr unterschiedliche Arten und Weisen beschrieben worden, beispielsweise als politischer Stil (Moffitt 2016), als politische Logik (Laclau 2005) oder als dünne politische Ideologie (Mudde 2004). Diese Studie schließt sich dem zuletzt genannten Ansatz an, der in der gegenwärtigen Populismusforschung als Konsens gehandelt wird (Hawkins et al. 2019; Wirth et al. 2016). Demnach handelt es sich bei Populismus nicht um eine besonders umfangreiche Ideologie, sondern vielmehr um ein grobes Gerüst aus wenigen Kernideen, welches sich mit einer „Wirtsideologie“" verbinden lässt (Freeden 1998; Mudde 2004). Diese Offenheit ermöglicht es Populismus, sich mit anderen Ideologien zu verbinden (z. B. Sozialismus, Ökologismus, Nationalismus etc.) und so sein „leeres Herz" mit verschiedenen ideologischen Werten zu füllen (Taggart 2004, S. 275; Mudde 2004, S. 544; Priester 2011, S. 3). Vor dem Hintergrund dieser Anpassungsgabe haben einigen Autoren Populismus als eine „dünne Ideologie“ (thin ideology) bezeichnet, welche kein substanzielles Wertesystem besitzt (Albertazzi und McDonnell 2008; Freeden 1998; Mudde 2004; Reinemann et al. 2017).

Von dieser Begriffsdefinition ausgehend, haben Wirth und Kollegen 2016 in Anlehnung an Mudde (2004) folgende Kernideen in der dünnen populistischen Ideologie identifiziert: Die populistische Ideologie sieht die Gesellschaft unvereinbar gespalten in zwei in sich jeweils homogene Gruppen. Auf der einen Seite steht die böse Elite. Auf der anderen Seite das gute und tugendhafte Volk. Diese beiden Gruppen konkurrieren um die Entscheidungsgewalt. Populismus beklagt, dass diese Gewalt in den Händen der politischen Elite liegt, wobei sie ihren rechtmäßigen Platz in den Händen des Volkes haben sollte. Populismus hat das Anliegen, die Souveränität des Volkes wiederherzustellen. Aus diesen Erörterungen lassen sich drei wesentliche Dimensionen herauslösen, die gemeinsam Populismus abbilden: Volksnähe, Elitenkritik und Wiederherstellung der Volkssouveränität. Da dünner Populismus in seiner Reinform in der politischen Realität äußerst selten vorkommt, haben eini- 
ge Autoren in der Auseinandersetzung mit dem Phänomen Rechtspopulismus eine weitere Dimension, die Exklusion, in ihren Populismusbegriff integriert (Hameleers et al. 2017b; Hameleers und de Vreese 2020). Steht Linkspopulismus im Zentrum der Untersuchungen, wird die politische Elite häufig um Wirtschaftseliten ergänzt (Wirth et al. 2016).

Die vorliegende Untersuchung zielt darauf ab, Populismus im bundesdeutschen Kontext zu untersuchen. Da die AfD in Deutschland im rechtspolitischen Spektrum verortet wird (Bebnowski 2015; Ceyhan 2016), soll auch der Populismusbegriff dieses Beitrags um die Exklusionsdimension ergänzt werden. Im Folgenden sollen die vier Dimensionen näher ausgeführt werden.

Volksnähe Das Volk ist der Dreh- und Angelpunkt der populistischen Ideologie. Es wird als homogene oder monolithische soziale Einheit beschrieben (Wirth et al. 2016, S. 10). Diese Betrachtung des Volkes, dessen Mitglieder alle die gleichen Eigenschaften, Interessen und Bedürfnisse besitzen, steht im Gegensatz zu einem pluralistischen, modernen Gesellschaftsbild (Mudde 2004, S. 544; Rensmann 2006, S. 63-64). Das Volk wird darüber hinaus idealisiert, indem ihm exklusive, positive Tugenden zugeschrieben werden. Dazu gehört beispielsweise der gesunde Menschenverstand, der, gemäß der populistischen Ideologie, das moralische Urteilen und Handeln des Volkes anleitet. Der Begriff Volk kann je nach populistischer Bewegung anders interpretiert werden und bleibt grundsätzlich vage.

Elitenkritik Die Elite bildet in der populistischen Ideologie den Gegenpol zum Volk. Nicht nur ist die Beziehung zwischen Elite und Volk durch unüberwindbare Differenzen definiert, sondern auch die normative Vorstellung von Volk und Elite kann als äußerst gegensätzlich verstanden werden. Populisten verfolgen eine „schwarz-weiß“ Sicht, die das Volk als sinnhaft gut darstellt und die Elite als böse, schlecht und verdorben (Meyer 2006, S. 82). Laut Populisten handelt die Elite gegen das Gemeinwohl, bedient in erster Linie ihre Eigeninteressen und bereichert sich auf Kosten des Volkes. Aus diesen Gründen ist die politische Elite auch verantwortlich für die als oft prekär gezeichnete Lage des Volkes. Der politischen Elite wird der Missbrauch des repräsentativen Amtes vorgeworfen, der mit einer Beschädigung der Gesellschaft und Demokratie einhergeht (Wirth et al. 2016, S. 11-12). Medien werden dabei oftmals als Komplizen der Elite verstanden, die dem Volk die Wahrheit verheimlichen oder es bewusst täuschen. Entsprechend wird Kritik an Medien geübt (Schindler et al. 2018).

Wiederherstellung der Volkssouveränität Das zentrale Motiv der populistischen Ideologie besteht darin, die Souveränität des Volkes wiederherzustellen. ,The central populist message is that politics has escaped popular control and that popular control has to be restored" (Kriesi 2014, S. 363). Das Zitat verdeutlicht die Kritik der Populisten am Status Quo des politischen Systems und zeigt gleichzeitig die Lösung für diesen Missstand auf: Die Macht ist in die Hände des Volkes zu geben. Aus Sicht der Populisten soll sich die Politik gänzlich durch den Volkswillen formen. Konstitutionelle Elemente der liberalen Demokratie, wie die der Gewaltenteilung oder der Schutz von Minderheiten werden durch derartige Forderungen implizit oder 
explizit in Frage gestellt, da diese den Volkswillen potentiell einschränken können (Kriesi 2014, S. 363). Mudde verwendet in diesem Zusammenhang den Begriff der ,illiberalen Demokratie“ oder auch den des ,demokratischen Extremismus“ (2004, S. 561).

Fremdenfeindlichkeit - Schaffen einer Outgroup Im Rechtspopulismus werden neben der politischen Elite auch Ausländer, Asylsuchende oder Immigranten als Gefahr für das Volk herausgestellt. In der Literatur ist hier oft die Rede von den sogenannten „Anderen“, die das Volk von unten bedrohen, wohingegen die politische Elite das Volk von oben bedroht (Reinemann et al. 2017). Die Konstruktion der bösen Anderen als Out-Group ist im Rahmen populistischer Kommunikation ein bewährtes Mittel, um das Volk weiter zu idealisieren und als gänzlich ,gut und rein“" abzugrenzen.

\section{Methode}

Für die explorative Studie wurden insgesamt 224 Initialbeiträge der Parteien AfD, CDU und SPD (AfD=74; $C D U=75 ; \mathrm{SPD}=75)$ und 448 Nutzerkommentare $(\mathrm{AfD}=148 ; \mathrm{CDU}=150 ; \mathrm{SPD}=150)$ von den offiziellen Facebook-Bundesseiten mittels einer standardisierten Inhaltsanalyse ausgewertet (Früh 2007; Rössler 2010). Standardisierte Inhaltsanalysen eignen sich besonders, um Textkörper auf das Vorhandensein bestimmter, vorab definierter, Kommunikationsmerkmale hin zu untersuchen. Dabei bringt die Methode der standardisierten Inhaltsanalyse unweigerlich eine Reduktion von Komplexität mit sich, wenn komplexe Texte durch ein kategorisches Raster analysiert werden (Rössler 2010, S. 19). Da es jedoch darum geht $\mathrm{zu}$ erfassen, ob Online-Kommunikation bestimmte populistische Merkmale aufweist und diese Merkmale adäquat aus der theoretischen Literatur abgeleitet und in disjunkte empirische Kategorien überführt werden können, ist diese Komplexitätsreduktion vertretbar.

Die Studie beschränkt sich dabei auf drei Parteien. Dabei wurde die AfD bewusst ausgewählt, weil dieser Partei immer wieder populistische Strategien unterstellt wurden. Die CDU und SPD wurden vor dem Hintergrund ihrer Rolle als Volksparteien ausgewählt. Dabei ist zu beachten, dass diese beiden Parteien im Zeitraum der Untersuchung eine Regierungskoalition bildeten. Somit ist die AfD die einzige Oppositionspartei, was bei der Interpretation der Ergebnisse zu berücksichtigen ist.

Für die Untersuchung wurden alle Posts und Nutzerkommentare der drei Parteien im Zeitraum von August 2016 bis Februar 2017 als Grundgesamtheit definiert und durch Screenshots archiviert. Durch den gewählten Untersuchungszeitraum von sieben Monaten sollten Verzerrungseffekte durch Schlüsselereignisse (Rauchenzauner 2008) minimiert werden. Es sollte vor allem die politische Kommunikation in der 
Normalzeit (Wolling 2005) analysiert werden. ${ }^{2}$ Im festgelegten Untersuchungszeitraum veröffentlichte die AfD insgesamt 395, die CDU 270 und die SPD insgesamt 334 Beiträge auf Facebook $(N=999)$. Die Auswahl der Initialposts erfolgte quasirandomisiert. So wurde bei der AfD jeder fünfte Initialpost, bei der CDU jeder dritte und bei der SPD jeder vierte Initialpost in das Sample aufgenommen $(n=224)$. Für die gesampelten Initialposts wurden im nächsten Schritt die entsprechenden Nutzerkommentare archiviert $(N=74.072)$. Jedem Initialpost wurden dann zwei Nutzerkommentare quasi-randomisiert zugewiesen, indem bei der CDU und der SPD jeder zehnte Kommentar, bei der AfD, auf Grund der deutlich höheren durchschnittlichen Menge an Kommentaren pro Post, jeder 20. Kommentar ausgewählt wurde $(n=448) .{ }^{3}$ Die ausgewählten Posts bzw. Kommentare wurden anschließend durch zwei geschulte Codierer inhaltsanalytisch verschlüsselt. Die Analyseeinheit war dabei der jeweilige Post bzw. Kommentar.

Operationalisierung Volksnähe Drei wesentliche Bestandteile prägen diese Dimension: (1) das Verständnis des „Volkes“ als homogene Gruppe, (2) die Zuschreibung positiver Attribute an das Volk und (3) die Identifikation des populistischen Akteurs mit dem Volk. Die Variable Homogenisierung misst, ob die Adressaten der Initialbeiträge/Kommentare als homogene Masse angesprochen werden (Wirth et al. 2016, S. 10). Kennzeichnend hierfür ist die Verwendung der Wörter „Volk“, „Nation“, „Deutsche“, „Bürger“ etc. Die Variable Idealisierung beschreibt, inwiefern Eigenschaften des Volkes als positiv dargestellt werden. Sämtliche positive Attribute und in der Literatur hervorgehobene Begriffe, wie der außerordentliche Gemeinschaftssinn, der gesunde Menschenverstand und die Attribute „einfach“ und „hart arbeitend“, fallen in diese Kategorie (Mudde 2004, S. 547; Priester 2012, S. 4). Darüber hinaus misst die Variable Selbstpositionierung, ob der politische Akteur seine Rolle in der Tradition des populistischen Beziehungskonzepts näher beschreibt, sich also entweder als Advokat oder Teil des Volkes positioniert und so versucht, eine Identifikation mit dem Volk zu evozieren (Wirth et al. 2016, S. 12, Schmuck und Hameleers 2019, S. 12). Da diese Variable an die Aussagen der politischen Akteure gebunden ist, wird sie nur auf Ebene der Initialposts erhoben.

Operationalisierung Elitenkritik Als Mitglied der Elite werden hier sowohl Funktionseliten aus Politik, Wirtschaft und Justiz verstanden, als auch Leistungseliten, wie etwa Prominente, die als politische Sprecher auftreten. Werden Personen

\footnotetext{
2 Es wurde dem Umstand Rechnung getragen, dass im gewählten Untersuchungszeitraum Landtagswahlen in Mecklenburg-Vorpommern (4. September) und in Berlin (18. September) stattfanden. Um entsprechende Verzerrungen zu minimieren wurden Parteien-Posts, die die Landtagswahlen thematisierten, ausgeschlossen und durch den nächsten Post ersetzt. Da es sich jedoch um die Bundesseiten der Parteien handelte, war dies nur in fünf Fällen (Wahlaufrufe) gegeben. Dennoch kann nicht gänzlich ausgeschlossen werden, dass die Landtagswahlkämpfe einen Einfluss auf die Online-Kommunikation auf Bundesebene genommen hat (dazu: Sarcinelli 2011, Kap. 12).

3 Dabei ist zu berücksichtigen, dass die Sortierung der Nutzerkommentare auf den drei untersuchten Seiten unterschiedlich war. Auf den Seiten von CDU und SPD wurden die Kommentare nach Beliebtheit (,,TopKommentare") angezeigt, während bei der AfD eine chronologische Kommentar-Anzeige vorlag. Dieser Umstand muss bei der Interpretation der Ergebnisse berücksichtigt werden.
} 
aus diesem Kreis benannt oder kollektiv angesprochen (,die da Oben“), wurde Elitenkritik geprüft (Mudde und Kaltwasser 2017, S. 12). Die negative Einstellung gegenüber der Elite kann innerhalb der populistischen Kommunikation mittels drei verschiedener Strategien zum Ausdruck kommen. Zum einen misst die Variable Kompetenzkritik, inwiefern fachliche Kompetenzen oder persönliche Eigenschaften einzelner Akteure, die der Elite angehören, bemängelt werden. Die Variable Moralkritik geht darüber hinaus explizit auf den Gemeinschaftssinn und die Gemeinwohlorientierung ein und trägt ab, ob der Elite unterstellt wird, ihre Eigeninteressen über das Gemeinwohl zu stellen. Die Variable Schuldzuweisung geht noch einen Schritt weiter und erfasst, ob die Elite für Missstände verantwortlich gemacht wird (Decker 2004, S. 36). Die vierte Variable der Populismusdimension, Medienkritik, folgt einer anderen Logik: Sie misst, ob Medien, als spezielle Gruppe der Elite, Adressat der Kritik werden (Jagers und Walgrave 2007).

Operationalisierung Wiederherstellung der Volkssouveränität Die Forderung nach Volkssouveränität kann auf zwei Wegen kommuniziert werden: Zum einen, indem mehr Kompetenzen und Macht für das Volk eingefordert werden oder indem der Elite im Umkehrschluss Kompetenzen und Macht abgesprochen werden (Wirth et al. 2016, S. 42). Die Dimension Wiederherstellung der Volkssouveränität beinhaltet folglich die zwei Variablen: Forderung Kompetenzen für das Volk und Absprache der Kompetenzen der Elite. Dabei wurde bei Begriffen wie „Volk“, „die Bürger“, „die Menschen“, „Gesellschaft“ oder auch „,wir“ geprüft, ob der Kontext des Kommentars eine Wiederherstellung der Volkssouveränität nahelegt (Wirth et al. 2016, S. 42).

Operationalisierung Fremdenfeindlichkeit Das Aufgreifen einer Outgroup richtet sich auf eine Gruppe, die auf ethnischen Unterschieden, also Herkunft, Religion und Kultur basiert (Taggart 2004, S. 270-271). Kommunikativ ist es möglich, sich von einer anderen gesellschaftlichen Gruppe abzugrenzen, in der Unterschiede zu dieser thematisiert werden (Winkler 2013, S. 34). Eine weitere Möglichkeit besteht darin, diese Gruppe für persönliche oder gesellschaftliche Missstände verantwortlich zu machen (Lochoki 2012, S. 32) oder diese zu beleidigen. Um diese Inhalte systematisch zu erfassen, wurden die Variablen Unterschied, Schuldzuweisung und Beleidigung gebildet.

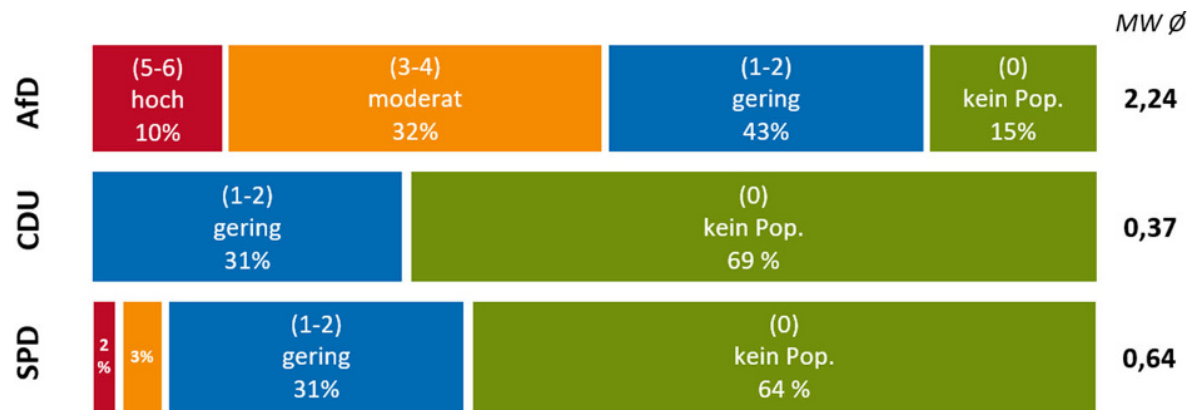

Abb. 1 Gehalt an Populismus in Initialposts pro Partei ohne Fremdenfeindlichkeit (Summenindex) 
Opposition Mit der Variable Opposition wird auf Ebene der Nutzerkommentare erhoben, welche Einstellungen die Nutzer gegenüber den im Sample vertretenen Parteien haben. Diese Variable soll abtragen, ob sich in den jeweiligen Kommentarbereichen Parteianhänger oder -kitiker zu Wort melden. Sofern dies offensichtlich ist, sollen Inhalte von parteikritischen Nutzern gesondert betrachtet werden.

Für die vorliegende Untersuchung wurde Populismus entlang der drei oben beschriebenen Dimensionen über insgesamt neun Variablen gemessen. Für alle neun codierten Populismus-Variablen sowie die drei Variablen zur Dimension der Fremdenfeindlichkeit lag die Codierübereinstimmung nach Holsti (1969) deutlich über dem kritischen Wert 0,70 (Reliabilität nach Holsti: $\left.C_{R}=0,89-0,98 ; \varnothing=0,95\right) .{ }^{4}$

Aus den neun Populismus-Variablen wurde ein Summenindex gebildet, um die Intensität populistischer Merkmale in den Posts und Kommentaren qualifiziert abbilden zu können. Dabei kann der Index theoretisch einen Wert zwischen 0-9 annehmen - empirisch konnten Werte zwischen 0 und 6 nachgewiesen werden. Die Dimension Fremdenfeindlichkeit mit insgesamt drei Variablen floss nicht in die Berechnung eines Populismusindexes ein, sondern wurde stets isoliert betrachtet. Um Populismus innerhalb der jeweiligen Posts bzw. Kommentare qualifizieren zu können, wurden schließlich vier Kategorien gebildet: kein Populismus (Indexwert=0); geringer Populismus (Indexwert $=1-2$ ); moderater Populismus (Indexwert $=3-4$ ) und hoher Populismus (Indexwert=5-6) (Abb. 1). Die Messung von Populismus mit Hilfe eines Indizes erlaubt es, differenzierte Aussagen darüber machen zu können, wie viele aus der Theorie abgeleitete Populismusindikatoren in Postings der Parteien bzw. in Nutzerkommentaren präsent sind und ist somit näher am theoretischen Populismuskonzept als die kategorische Einteilung in populistische (1) und nicht populistische (0) Kommunikation, wie es etwa Ernst et al. (2017) vorschlagen.

\section{Ergebnisse}

\subsection{Populismus in der Parteienkommunikation}

Die deskriptive Auswertung der Initialposts zeigt, dass der Anteil populistischer Aussagen in den Posts der AfD am höchsten ist: Rund 85\% der AfD-Posts enthalten populistische Elemente. Bei SPD (36\%) und CDU (31\%) sind wesentlich seltener populistische Merkmale vorhanden.

Um die Intensität populistischer Inhalte zwischen den Parteien besser vergleichen zu können, wurde der Mittelwert des Summenindex für Populismus über alle Posts einer Partei hinweg berechnet. Die Dimension Fremdenfeindlichkeit wurde dabei außen vorgelassen. Der Mittelwert über alle Posts liegt bei der AfD $(M=2,24$; $S D=1,56)$ verglichen mit SPD $(M=0,64 ; S D=1,05)$ und CDU $(M=0,37 ; S D=0,61)$ am höchsten $(F(2,221)=76,05, p<0,001, \eta 2=0,342)$. Das bedeutet, dass im Durchschnitt jeder Post der AfD 2,24 Populismusindikatoren aufwies, wohingegen bei der

\footnotetext{
${ }^{4}$ Codierübereinstimmung nach Holsti: $\mathrm{C}_{\mathrm{R}}=2 * \mathrm{C}_{\mathrm{U}} /\left(\mathrm{C}_{\mathrm{A}}+\mathrm{C}_{\mathrm{B}}\right)$; Erläuterung: $\mathrm{C}_{\mathrm{R}}=$ Reliabilitätskoeffizient der Codierung; $\mathrm{C}_{U}=$ Zahl der Übereinstimmenden Codierungen; $\mathrm{C}_{\mathrm{A}}=$ Zahl der Codierungen Codierer A; $\mathrm{C}_{\mathrm{B}}=$ Zahl der Codierungen Codierer B (Rössler 2010, S. 202).
} 
Tab. 1 Ausprägung der Variablen für Parteiposts und Nutzerkommentare (in Prozent)

\begin{tabular}{|c|c|c|c|c|c|c|c|}
\hline \multirow[t]{2}{*}{ Dimension } & \multirow[t]{2}{*}{ Variable } & \multicolumn{2}{|c|}{ Post der Parteien } & \multicolumn{4}{|c|}{ Nutzerkommentare } \\
\hline & & AfD & $\mathrm{CDU}$ & SPD & AfD & $\mathrm{CDU}$ & SPD \\
\hline \multirow[t]{3}{*}{ Volksnähe } & Homogenität & 45 & 17 & 20 & 16 & 25 & 14 \\
\hline & Idealisierung & 5 & 3 & 7 & 0 & 5 & 1 \\
\hline & Selbstpositionierung & 8 & 5 & 5 & - & - & - \\
\hline \multirow[t]{4}{*}{ Elitenkritik } & Kompetenzkritik & 39 & 4 & 3 & 17 & 29 & 27 \\
\hline & Moralkritik & 62 & 5 & 8 & 37 & 53 & 45 \\
\hline & Schuldzuweisung & 36 & 0 & 5 & 23 & 37 & 17 \\
\hline & Medienkritik & 12 & 1 & 0 & 7 & 3 & 1 \\
\hline \multirow{2}{*}{$\begin{array}{l}\text { Wiederherstellung } \\
\text { Volkssouverä- } \\
\text { nität }\end{array}$} & Absprache & 15 & 0 & 0 & 2 & 1 & 1 \\
\hline & Forderung & 9 & 1 & 16 & 9 & 8 & 1 \\
\hline \multirow{5}{*}{$\begin{array}{l}\text { Fremdenfeind- } \\
\text { lichkeit }\end{array}$} & Unterschiede & 28 & 3 & 0 & 17 & 7 & 2 \\
\hline & Schuldzuweisung & 19 & 0 & 0 & 7 & 8 & 3 \\
\hline & Beleidigung & 3 & 0 & 0 & 5 & 0 & 0 \\
\hline & & $N=74$ & $N=75$ & $N=75$ & $N=148$ & $N=150$ & $N=150$ \\
\hline & & $n=224$ & & & $n=448$ & & \\
\hline
\end{tabular}

SPD 0,64 und bei der CDU 0,37 Indikatoren pro Post präsent waren. Der Posthoc-Test nach Scheffé zeigt sowohl, dass sich die CDU signifikant von der AfD unterscheidet $(p<0,001)$, als auch, dass SPD und AfD sich signifikant voneinander unterscheiden $(p<0,001)$. Die Unterschiede zwischen CDU und SPD sind nicht signifikant $(p=0,463)$.

Die AfD postet dabei nicht nur anteilsmäßig mehr populistische Initialbeiträge auf ihrer offiziellen Facebook-Seite als CDU und SPD. Die AfD-Posts neigen auch dazu, gleichzeitig mehrere Populismusdimensionen (Volksnähe, Elitenkritik, Wiederherstellung Volkssouveränität) anzusprechen, als es bei CDU und SPD der Fall ist. Während bei der CDU und SPD in $31 \%$ der Fälle ein geringer Populismuswert ermittelt wurde (1-2 Indikatoren), sind bei der AfD bei $42 \%$ aller Posts moderate bis hohe Populismuswerte zu verzeichnen (3-6 Indikatoren). Lediglich 15\% der AfDPosts weisen keine Merkmale von Populismus auf, wohingegen rund zwei Drittel der Posts von CDU und SPD keinen populistischen Indikator beinhalten (Abb. 1).

Betrachtet man die Teildimensionen (Tab. 1), wird deutlich, dass Volksnähe Bestandteil der Kommunikation aller untersuchten Parteien ist. Allerdings hebt die AfD mit $45 \%$ wesentlich häufiger auf die Homogenität des Volkes ab, als es CDU (17\%) und SPD (20\%) tun. Zudem sind die Dimensionen Elitenkritik und Fremdenfeindlichkeit fast ausschließlich Bestandteil der AfD-Kommunikation. So wird etwa in $62 \%$ der AfD-Posts moralisch Kritik an der Elite geübt, wohingegen diese Kritikform bei der SPD (8\%) und der CDU (5\%) kaum eine Rolle spielt. Kompetenzkritik wird ebenfalls deutlich häufiger von der AfD (39\%) formuliert, als von CDU (4\%) und SPD (3\%). Schließlich wird die Medienkritik nahezu exklusiv von der AfD formuliert (12\%). In $37 \%$ der AfD Posts lässt sich mindestens eine Dimension von Fremdenfeindlichkeit finden, mehr als zehnmal so viel verglichen mit der CDU (3\%). In der Kategorie Fremdenfeindlichkeit macht die AfD vor allem Unterschiede salient (28\%) oder formuliert Schuldzuweisungen (19\%), wohingegen 


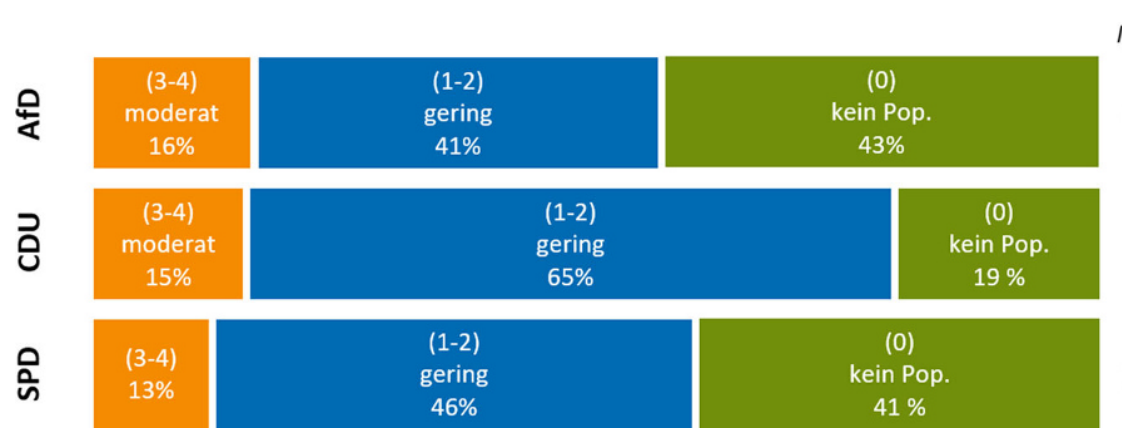

$M W \varnothing$

Abb. 2 Gehalt an Populismus in Kommentaren pro Partei ohne Fremdenfeindlichkeit (Summenindex)

Beleidigungen nur selten (3\%) vorkommen. Über alle Parteien hinweg spielt Wiederherstellung der Volkssouveränität eine untergeordnete Rolle. Dabei fällt jedoch auf, dass die AfD als einzige Partei der Elite die Führungskompetenz abspricht (15\%). Die Forderung nach mehr Volkskompetenzen wird hingegen am häufigsten von der SPD $(16 \%)$ formuliert.

\subsection{Populismus in der Anschlusskommunikation}

Nachdem auf der Ebene der Parteienkommunikation (Initialposts) die AfD die meisten populistischen Inhalte kommuniziert hat, zeigt die Analyse der Nutzerkommentare ein anderes Ergebnis: Nutzerkommentare auf der Facebook-Seite der CDU weisen in $81 \%$ der Fälle populistische Indikatoren auf. Mit einigem Abstand folgen dann die SPD (59\%) und die AfD (57\%). Die Nutzer-Kommentare auf der CDU-Seite verzeichnen auch in Sachen Populismusintensität den höchsten Mittelwert $(M=1,60$; $S D=1,16)$, gefolgt von der $\operatorname{AfD}(M=1,11 ; S D=1,22)$ und der $\operatorname{SPD}(M=1,08$; $S D=1,14)$ (Abb. 2). Die Mittelwertunterschiede sind signifikant $(F(2 / 445)=9,26$, $p<0,001, \eta 2=0,04)$. Der durchgeführte Post-hoc-Test nach Scheffé zeigt, dass sich die Signifikanz der Mittelwertunterschiede auf die Unterschiede zwischen CDU und SPD $(p<0,001)$ einerseits und CDU und AfD $(p<0,01)$ andererseits zurückführen lassen. Zwischen AfD und SPD sind keine signifikanten Unterschiede zu finden $(p=0,977)$. Betrachtet man die Mittelwerte, sind die Nutzerkommentare auf CDU und SPD Facebook-Seiten im Vergleich zu den eigenen Posts populistischer. Hingegen sind die Nutzerkommentare auf der AfD Facebook-Seite vergleichsweise weniger populistisch als die Initialposts der Partei.

Schaut man sich die Dimensionen von Populismus im Detail an, finden sich auf der CDU-Seite die höchsten Werte in den Dimensionen Volksnähe und Elitenkritik. Über die Hälfte der CDU-Nutzerkommentare (53\%) weißen Moralkritik auf. Die AfD-Nutzerkommentare liegen hier mit $37 \%$ deutlich hinter denen der SPD (45\%). Auch bei der Kompetenzkritik liegen die Nutzerkommentare auf der CDU-Seite (29\%) vor denen auf der SPD- (27\%) und AfD-Seite (17\%). Mit Blick auf die Volksnähe verweist jeder vierte CDU-Nutzerkommentar auf die Homogenität, was Kommentare auf der AfD-Seite lediglich in $16 \%$ der analysierten Fälle tun. Wird die Dimension Fremdenfeindlichkeit jedoch näher betrachtet, zeigt sich, dass $23 \%$ der 
Nutzerkommentare bei der AfD fremdenfeindliche Elemente aufweisen, gefolgt von CDU (11\%) und SPD (4\%). Tab. 1 zeigt die einzelnen Ausprägungen der jeweiligen Variablen für die Parteienposts und die Nutzerkommentare.

Um zu prüfen, ob der Populismusgehalt in den Initialposts der Parteien einen Einfluss auf den Populismusgehalt in den Nutzerkommentaren hat, wurde eine zweiseitige Pearson Korrelation berechnet. Die Analyse offenbart über alle Kommentare hinweg vorerst keine signifikanten Ergebnisse $(p=0,08) .{ }^{5}$ Betrachtet man den Zusammenhang zwischen dem Populismusgehalt der Initialposts und dem Populismusgehalt der Nutzerkommentare gesondert pro Partei, wird deutlich, dass ein positiver Zusammenhang sowohl auf der Seite der CDU $(r=0,264 ; p<0,05)$ als auch der AfD $(r=0,247 ; p<0,05)$ besteht. Bei der SPD hingegen lässt sich kein signifikanter Zusammenhang feststellen ( $p=0,249)$.

Schließlich zeigt die Analyse Unterschiede in der oppositionellen Nutzerstruktur. Von den insgesamt 448 untersuchten Nutzerkommentaren wurden rund $21 \%$ als oppositionell codiert. Das bedeutet, dass etwa jeder fünfte Kommentar sich kritisch zu der Partei äußert, auf dessen Seite der Kommentar abgeben wurde. Betrachtet man die Verteilung dieser oppositionellen Kommentare, zeigt sich, dass auf der CDU-Seite mit rund $41 \%$ die mit Abstand meisten oppositionellen Kommentare zu finden sind. Bei der SPD ist etwa jeder fünfte Kommentar (19\%) oppositionell, wohingegen bei der AfD lediglich etwa $5 \%$ kritisch gegenüber der AfD sind. Dieser Befund deutet entweder auf eine sehr unterschiedliche Nutzerstruktur auf den jeweiligen Parteiseiten hin oder aber auf Unterschiede im Bereich des Community-Managements. Eine nähere Analyse der oppositionellen Kommentare zeigt, dass diese deutlich höhere Populismuswerte aufweisen. Die Mittelwerte der PopulismusIndizes unterscheiden sich signifikant. Während nicht-oppositionelle Kommentare einen Mittelwert von 1,08, $(S D=1,13)$ aufweisen, liegt der Mittelwert bei oppositionellen Kommentaren bei $1,91(S D=1,23)(r=0,865 ; p<0,00)$.

\section{Diskussion}

Ziel der vorliegenden Untersuchung war zu prüfen, inwieweit sich Parteien in ihren Facebook-Posts eines populistischen Kommunikationsstils bedienen und dabei Unterschiede zwischen der AfD, der CDU und der SPD zu analysieren. Zudem wurde erstmals die Anschlusskommunikation in Form der Nutzerkommentare unter den Parteiposts in Hinblick auf ihren Populismusgehalt untersucht und exploriert, ob es einen Zusammenhang zwischen dem Populismusgehalt in den Parteienposts und den darauffolgenden Nutzerkommentaren gibt. Die Anschlusskommunikation ist dabei von besonderem Interesse, weil es sich dabei gewissermaßen um einen von Parteien organisierten und über die Postings stimulierte Debatte handelt. $\mathrm{Ob}$ ihrer hervorgehobenen Stellung im politischen System der Bundesrepublik kommt Parteien eine besondere Rolle im Prozess politischer Kommunikation zu, was eine entsprechende Analyse parteilich initiierter Online-Diskussionen begründet. Um Po-

\footnotetext{
5 Die einseitige Pearson-Korrelation offenbart zwar einen signifikanten positiven Zusammenhang, der jedoch nur schwachen ausfällt $(r=0,123, p<0,033)$.
} 
pulismus messbar zu machen, wurden die drei Dimensionen Volksnähe, Elitenkritik und Wiederherstellung der Volkssouveränität operationalisiert und um die Dimension Fremdenfeindlichkeit ergänzt. Im Folgenden sollen nun die Ergebnisse diskutiert und eingeordnet sowie Limitierungen der Studie offengelegt werden.

\subsection{Diskussion der Ergebnisse}

Die Analyse offenbart, dass die AfD populistische Inhalte mit Abstand sowohl häufiger als auch intensiver kommuniziert als CDU oder SPD. Dabei fällt auf, dass die AfD sich vor allem an bestehenden Eliten und dem vermeintlich Fremden kommunikativ abarbeitet: Die Dimensionen Elitenkritik und Fremdenfeindlichkeit sind fast ausschließlich bei der AfD vorzufinden. Dieses Ergebnis kann einerseits als erwartbar gelten, da die AfD gemeinhin als rechtspopulistische Partei eingestuft wird (Bebnowski 2015; Ceyhan 2016). Es ist allerdings zu beachten, dass sich die hohe Ausprägung der Elitenkritik im Falle der AfD auch aus ihrer Oppositionsrolle heraus erklären kann. So fanden beispielsweise Heiss und Matthes (2019), dass auch die liberale österreichische Oppositionspartei NEOS häufig anti-elitistische Statements auf Facebook teilte. Auch andere Studien wiesen höhere Populismuswerte für die Kommunikation von Oppositionspartien im Vergleich zu Regierungsparteien nach. Allerdings waren es immer die populistischen Oppositionsparteien, insbesondere die am rechten Rand, die die höchsten Populismuswerte aufwiesen (Jagers und Walgrave 2007; Ernst et al. 2017; Engesser et al. 2017).

Die Ergebnisse deuten darauf hin, dass soziale Medien nicht per se zu einer populistischen Parteienkommunikation führen müssen, denn sowohl bei CDU als auch bei SPD finden sich vergleichsweise selten $(31 \%$ \& 36\%) und wenn nur sehr wenige populistische Indikatoren (1-2). Die als populistisch einzustufende AfD nutzt die ihnen zu Verfügung stehenden Kanäle hingegen dafür, populistische Botschaften auszusenden. So finden sich in $85 \%$ aller Posts Indikatoren für Populismus, wobei $42 \%$ zwischen 3-6 Populismusindikatoren aufweisen. Medienkritik und Fremdenfeindlichkeit sind dabei fast ausschließlich bei der AfD anzutreffen. Vor dem Hintergrund der im Vergleich höchsten Distributionsrate der AfD-Posts ( $\varnothing$ Shares pro Post: AfD=3504; SPD=876; CDU =335), kann die Partei somit eine hohe Reichweite (rechts)populistischer Inhalte über Facebook erzielen (dazu auch: Staender et al. 2019).

Während der nachgewiesene Populismusgehalt für die Parteienposts im Bereich des Erwartbaren liegt, sind die Ergebnisse mit Blick auf die Anschlusskommunikation in den Nutzerkommentaren durchaus als überraschend einzustufen. Die Nutzerkommentare auf der CDU-Seite sind hier mit Abstand am populistischsten. Eine Erklärung dafür könnte die aktive Nutzerschaft der AfD sein, die bei konservativen Kontrahenten versucht ,,populistische Stimmung“ zu machen. Ergebnisse einer Studie von Stier et al. (2017) stärken diese Annahme. Das Forscherteam fand heraus, dass die Kommentarfunktion von Nutzern vorwiegend als Werkzeug genutzt wird, um der eigenen Kritik an Parteien und Politik Ausdruck zu verleihen, wohingegen Likes als Instrument der Zustimmung dienen. Zudem sprechen die Auswertungen der User-Aktivität durch Web-Tracking-Daten dafür, dass AfD-Sympathisanten auch auf CDU-Seiten aktiv sind, wo sie Inhalte der CDU häufig (kritisch) kommentieren 
(Stier et al. 2017, S. 1373-1374). Da die vorliegende Untersuchung auch gezeigt hat, dass $41 \%$ der CDU-Nutzerkommentare kritisch gegenüber der CDU sind und diese oppositionellen Kommentare signifikant populistischer sind als nicht-oppositionelle Kommentare, erscheint es nicht unplausibel, dass hier kommunikative Opposition betrieben wurde und AfD-Sympathisanten möglicherweise systematisch auf der Seite des politischen Gegners (hier CDU) populistische Botschaften senden. Gleichsam deutet die geringe kommunikative Opposition auf der AfD-Seite darauf hin, dass kritische Stimmen hier entweder gar nicht auftreten oder aber von Administratoren gelöscht werden. Dies kann an dieser Stelle jedoch nur spekuliert und nicht empirisch belegt werden. Eine alternative Erklärung liefert die Regierungsrolle der CDU. Regierungsparteien bieten Angriffsfläche für Elitenkritik, die in den CDUNutzerkommentaren am stärksten ausgeprägt ist und entsprechend auf eine zentrale Populismusdimension einzahlt.

Mit Blick auf die Frage, inwieweit Populismus in Parteienposts den Populismusgehalt in Nutzerkommentaren beeinflusst, liefert die Untersuchung keine eindeutigen Ergebnisse. Über alle drei untersuchten Parteien hinweg, zeigt sich kein signifikanter Zusammenhang zwischen dem Populismusgehalt in den Initialposts der Parteien und dem Populismusgehalt in den Nutzerkommentaren. Betrachtet man allerdings die Zusammenhänge für jede Partei gesondert, zeigt sich ein signifikanter Zusammenhang für AfD und CDU, jedoch nicht für die SPD. Wenn populistische Kommunikation eine entsprechende Wirkung hat, sollte sich diese gleichermaßen über alle untersuchten Partien hinweg zeigen, unabhängig vom durchschnittlichen Populismusgehalt in Posts und Kommentaren. Der fehlende Zusammenhang zwischen Populismus in Initialpost und Populismus in der Anschlusskommunikation auf der SPD-Facebook Seite ist mit den vorliegenden Daten nur schwer zu erklären. Es ist möglich, dass entsprechende Kommentare auf der SPD-Seite anders moderiert werden als bei der CDU und der AfD. Wahrscheinlicher erscheint es aber, dass die SPD Facebook-Seite nicht die gleiche Angriffsfläche für den politischen Gegner bietet, wie die Facebook-Seite der CDU. Immerhin konnte gezeigt werden, dass der Großteil der populistischen Nutzerkommentare auf der CDU-Seite oppositioneller Natur war. Wenn dem so ist, provoziert populistische Kommunikation von Regierungsparteien (hier CDU und SPD) eventuell nur populistische Kommunikation der politischen Gegner, nicht aber der eigenen Parteianhänger. In diesem spezifischen Fall konzentrieren sich die Gegner auf die CDU, die die deutsche Regierung über die Kanzlerschaft eventuell stärker repräsentiert als der Koalitionspartner SPD. Ohne weitere Forschung lässt sich an dieser Stelle also lediglich festhalten, dass der Populismusgehalt in den von den Parteien verantworteten Posts nicht in jedem Fall zu mehr Populismus führt. Gleichzeitig liegen die hier erbrachten Befunde für die Facebook-Seiten der AfD und der CDU aber auf einer Linie mit der von Blassnig et al. (2019b) gemachten Beobachtung, dass Populismus in Nachrichtenartikeln populistische Nutzerkommentare nach sich zieht.

Vor dem Hintergrund dieser Ergebnisse und der zentralen Rolle von Parteien bei der Stiftung politischer Debatten, sollte künftige Forschung diese parteiinitiierten Diskussionen stärker und umfangreicher in den Blick nehmen, als dies hier geleistet werden konnte. Soziale Medien stellen dafür einen vielversprechenden Beobachtungsraum dar. Um den Zusammenhang populistischer Initialkommunikation 
auf die Anschlusskommunikation noch zielgerichteter untersuchen zu können, sollte künftige Forschung auch auf quasiexperimentelle Designs zurückgreifen, in denen der Populismusgehalt des Stimulus (etwa Postings) kontrolliert werden kann.

\subsection{Limitationen}

Die vorliegende Studie unterliegt einigen Limitierungen, die bei der Einordnung der Ergebnisse berücksichtigt werden müssen. Einige davon sind auf forschungsökonomische Rahmenbedingungen zurück zu führen, andere auf theoretische und methodische Entscheidungen. Eine Limitierung besteht im Hinblick auf die Sampling-Prozedur und der daraus resultierenden Datengrundlage. Für die Inhaltsanalyse wurden lediglich Text-Posts der Parteien ausgewertet, wohingegen etwa Videos aus der Analyse ausgeschlossen wurden. Bei rund einem Fünftel der erhobenen Initialposts der CDU und etwas weniger als einem Drittel bei der SPD waren jedoch Videos eingebettet. Die Kommunikation der AfD hingegen beschränkte sich mit Ausnahme von einem Post vollständig auf Text und Bild. Es kann somit nicht ausgeschlossen werden, dass Parteien, die vermehrt audiovisuelles Material bereitstellen, sich gerade bei dieser Ausdrucksform populistischer Stilmittel bedienen.

Eine zusätzliche Limitierung des Samples betrifft die angesprochenen Unterschiede bei der Sortierung der Nutzerkommentare (Top-Kommentare CDU \& SPD vs. chronologisch AfD), die die Vergleichbarkeit der Kommentare erschweren. Gegen eine systematische Verzerrung spricht jedoch, dass die SPD trotz derselben Auflistung (Top-Kommentare) den geringsten populistischen Gehalt auf der Kommentarebene aufweist. Zudem zeigt eine vergleichbar angelegte Untersuchung, bei der die Kommentarsortierung über alle Gruppen konstant gehalten wurde, ähnliche Ergebnisse (Spieß 2020).

Die Limitierung lenkt gleichsam den Blick auf einen in dieser Untersuchung ausgeklammerten Faktor: die Rolle von Intermediären. Dabei sind in sozialen Netzwerken insbesondere Algorithmen von Interesse, die die Sortierung und Sichtbarkeit von Beiträgen steuern und deren detaillierte Funktionslogiken zu den gut gehüteten Geheimnissen der Plattformbetreiber zählen. Inwieweit populistische Inhalte in Algorithmen Berücksichtigung finden, ist eine weitgehend offene Frage (dazu: Gerbaudo 2018). Hingegen verdichten sich die Evidenzen, dass populistische Parteien diverse Taktiken nutzen, um ihre Reichweiten in sozialen Medien zu optimieren, beispielsweise durch Social Bots (Dittrich 2017). Ob und inwieweit der Einsatz solcher Tools die Ergebnisse der vorliegenden Untersuchung beeinflusst hat, muss ob des gewählten Designs hier unbeantwortet bleiben. Selbiges gilt für den Einfluss von Netzaktivismus (Ziegele et al. 2020) und individueller Nutzereigenschaften (Ferrucci et al. 2020).

Eine weitere Limitierung betrifft die geringe Anzahl und funktionale Heterogenität der untersuchten Parteien. Mit der AfD wurde eine als rechtspopulistisch einzustufende Partei ausgewählt, die bislang immer nur als Oppositionspartei ohne Regierungsbeteiligung fungierte (Bebnowski 2015; Ceyhan 2016). Mit der CDU als konservative und der SPD als linksliberale Partei wurden zwei historische Volksparteien analysiert, die zum Analysezeitpunkt in einer großen Kollation regierten. Somit ergeben sich zahlreiche Unterschiede, die ebenfalls erklärend für die Parteikommu- 
nikation sein können (etwa die die vermehrte Elitenkritik aus der Oppositionsrolle heraus). Eine nicht-populistische oder dezidiert linke oder gar links-populistische (Oppositions-)Partei als Gegenspieler zur rechts-populistischen AfD hätte das analytische Spektrum abgerundet. Im Rahmen zukünftiger Studien sollte eine höhere Anzahl an Parteien, die ein möglichst breites politisches Spektrum von links außen bis rechts außen abdecken, mit einbezogen werden, um besser zu bestimmen für wen und unter welchen Bedingungen populistische Kommunikation wahrscheinlicher wird.

In theoretischer Hinsicht ist kritisch anzumerken, dass die hier angewandte Operationalisierung von Populismus zwar im Feld etabliert (Schmuck und Hameleers 2019; Wettstein et al. 2018b; Wirth et al. 2016), aber nicht gänzlich unproblematisch ist. So finden sich eine Reihe von Merkmalen, die auch als typische demokratische Rhetorik klassifiziert werden könnten. So ist der Rekurs auf den Volkskörper durchaus plausibel, wenn Parteien auf demokratische Legitimität durch das Volk abzielen und auch der Gedanke, dass das Volk grundsätzlich souverän ist, ist im Kern demokratisch. Ebenso ist die Kritik an Eliten ein typischer Modus demokratischer Auseinandersetzung. Um diesem Umstand Rechnung zu tragen, wurden einfache Summenindizes genutzt, mit deren Hilfe dann Aussagen über die Häufung populistischer Merkmale und damit der Intensität populistischer Kommunikation gemacht werden konnten.

Schließlich ist anzumerken, dass die Datengrundlage für eine quantitativ angelegte Studie mit knapp 75 Facebook-Posts, sowie jeweils 150 Nutzerkommentaren pro Partei vergleichsweise klein ausfällt, was die Aussagekraft der Ergebnisse schmälert. Dennoch erlaubt die Fallzahl explorative Schlüsse, die in weiteren Studien mit einer umfangreicheren Datengrundlage erneut überprüft werden sollten.

\section{Schlussbemerkung}

Der vorliegende Beitrag hatte zum Ziel, den Populismusgehalt der Facebook-Kommunikation von Parteien sowie der auf den Parteiseiten stattfindenden Nutzerkommunikation zu bestimmen. Insbesondere die Anschlusskommunikation in Nutzerkommentaren wurde bisher selten auf ihren Populismusgehalt hin untersucht. Die Befunde lassen eine weitere und tiefergehende Auseinandersetzung mit Populismus in Nutzerkommentaren notwendig erscheinen, zumal sie neue Fragen aufwerfen: Werden Nutzer selbst zum strategischen Kommunikator und erzeugen sie gezielt (populistische) Gegenöffentlichkeiten online? Können diese Gegenöffentlichkeiten als populistische Echokammern beschrieben werden oder kommt es zu deliberativen oder agonistischen Episoden? Schließlich gilt es weiter zu erforschen, welche Rolle Parteien als strategische Organisationen bei diesen Prozessen spielen.

Danksagung Wir danken Isabelle Borucki und Dennis Michels für die wertvollen Hinweise und Anregungen im Vorfeld der Einreichung sowie den anonymen Gutachern für ihre kritischen und konstruktiven Kommentare

Funding Open Access funding provided by Projekt DEAL. 
Open Access Dieser Artikel wird unter der Creative Commons Namensnennung 4.0 International Lizenz veröffentlicht, welche die Nutzung, Vervielfältigung, Bearbeitung, Verbreitung und Wiedergabe in jeglichem Medium und Format erlaubt, sofern Sie den/die ursprünglichen Autor(en) und die Quelle ordnungsgemäß nennen, einen Link zur Creative Commons Lizenz beifügen und angeben, ob Änderungen vorgenommen wurden.

Die in diesem Artikel enthaltenen Bilder und sonstiges Drittmaterial unterliegen ebenfalls der genannten Creative Commons Lizenz, sofern sich aus der Abbildungslegende nichts anderes ergibt. Sofern das betreffende Material nicht unter der genannten Creative Commons Lizenz steht und die betreffende Handlung nicht nach gesetzlichen Vorschriften erlaubt ist, ist für die oben aufgeführten Weiterverwendungen des Materials die Einwilligung des jeweiligen Rechteinhabers einzuholen.

Weitere Details zur Lizenz entnehmen Sie bitte der Lizenzinformation auf http://creativecommons.org/ licenses/by/4.0/deed.de.

\section{Literatur}

Aalberg, Toril, Frank Esser, Carsten Reinemann, Jesper Stromback, und Claes de Vreese. 2017. Populist political communication in europe. London: Routledge.

Abts, Koen, und Stefan Rummens. 2007. Populism versus democracy. Political Studies 55(2):405-424.

Albertazzi, Daniele, und Duncan McDonnell (Hrsg.). 2008. Twenty-first century populism: Thespectre of Western European democracy. Basingstoke: Palgrave Macmillan.

Alemann, Ulrich, Philipp Erbentraut, und Jens Walther. 2018. Das Parteiensystem der Bundesrepublik Deutschland: Eine Einführung, 5. Aufl., Wiesbaden: Springer VS.

Bartlett, Jamie. 2014. Populism, social media and democratic strain. In Democracy in Britain: Essays in honour of James Cornford, Hrsg. Guy Lodge, Glenn Gottfried, 91-96. London: Institute for Public Policy Research.

Bebnowski, David. 2015. Die Alternative für Deutschland. Aufstieg und gesellschaftliche Repräsentanz einer rechten populistischen Partei. Wiesbaden: Springer VS.

Berbuir, Nicole, Marcel Lewandowsky, und Jasmin Siri. 2015. The AfD and its Sympathisers: finally a right-wing populist movement in Germany? German politics 24(2):154-178.

Bimber, Bruce. 1998. The Internet and political transformation: Populism, community, and accelerated pluralism. Polity 31(1):133-160.

Blassnig, Sina, Sven Engesser, Nicole Ernst, und Frank Esser. 2019b. Hitting a nerve: populist news articles lead to more frequent and more populist reader comments. Political Communication 36(4):629-651.

Blassnig, Sina, Nicole Ernst, Florin Büchel, und Sven Engesser. 2018. Populist communication in talk shows and social media: a comparative content analysis in four countries. SCM 7(3):338-363.

Blassnig, Sina, Nicole Ernst, Florin Büchel, Sven Engesser, und Frank Esser. 2019. Populism in Online election coverage. Journalism Studies 20(8):1110-1129.

Bos, Linda, und Kees Brants. 2014. Populist rhetoric in politics and media: A longitudinal study of the Netherlands. European Journal of Communication 29(6):703-719.

Bos, Linda, Wouter van der Brug, und Claes de Vreese. 2010. Media coverage of right-wing populist leaders. Communications 35(2):141-163.

Canovan, Margaret. 1981. Populism. New York: Harcourt Brace Jovanovich.

Canovan, Margaret. 1999. Trust the people! Populism and the two faces of democracy. Political Studies 47(1):2-16.

Ceyhan, Sara. 2016. Konservativ oder doch schon rechtspopulistisch? Die politischen Positionen der AfDParlamentskandidaten im Parteienvergleich. Zeitschrift für Politikwissenschaft 26(1):1-28.

Chadwick, Andrew, und Jennifer Stromer-Galley. 2016. Digital media, power, and democracy in parties and election campaigns. The International Journal of Press/Politics 21(3):283-293.

Decker, Frank. 2004. Der neue Rechtspopulismus. Opladen: Springer VS.

Dittrich, Paul-Jasper. 2017. Social networks and populism in the EU. Four things you should know. Jacques Delors Institut Policy Paper 192. Abrufbar am 15. Apr. 2020 unter: https://www.delorsinstitut.de/ 2015/wp-content/uploads/2017/04/20170419_SocialNetworksandPopulism-Dittrich.pdf

Donges, Patrick. 2008. Einleitung: Parteien als politische Organisationen in der Mediengesellschaft. In Medialisierung politischer Organisationen, Hrsg. Patrick Donges, 15-32. Wiesbaden: Springer VS.

Emmer, Martin. 2019. Online-Kommunikation politischer Akteure. In Handbuch Online-Kommunikation, Hrsg. Wolfgang Schweiger, Klaus Beck, 36-58. Wiesbaden: Springer VS. 
Engesser, Sven, Nicole Ernst, Frank Esser, und Florian Büchel. 2017. Populism and social media: How politicians spread a fragmented ideology. Information, Communication \& Society 20(8):1109-1126.

Ernst, Nicole, Sina Blassnig, Sven Engesser, Florin Büchel, und Frank Esser. 2019b. Populists prefer social media over talk shows: an analysis of populist messages and stylistic elements across six countries. Social Media and Society 5(1):1-14.

Ernst, Nicole, Sven Engesser, Florin Büchel, Sina Blassnig, und Frank Esser. 2017. Extreme parties and populism: an analysis of Facebook, and Twitter across six countries. Information, Communication \& Society 20(9):1347-1364.

Ernst, Nicole, Frank Esser, Sina Blassnig, und Sven Engesser. 2019a. Favorable opportunity structures for populist communication: comparing different types of politicians and issues in social media, television and the press. International Journal of Press/Politics 24(2):165-188.

Esser, Frank, Agnieszka Stepinska, und David Hopmann. 2016. Populism and the media. Cross-national findings and research perspectives. In Populist political communication in Europe, Hrsg. Toril Aalberg, Frank Esser, Carsten Reinemann, Jesper Strömbäck, und Claes de Vreese, 1-13. London: Routledge.

Ferrucci, Patrick, Toby Hopp, und Chris Vargo. 2020. Civic engagement, social capital, and ideological extremity: Exploring online political engagement and political expression on Facebook. New Media \& Society 22(6):1095-1115.

Freeden, Michael. 1998. Is nationalism a distinct ideology? Political Studies 46(3):748-765.

Früh, Werner. 2007. Inhaltsanalyse. Theorie und Praxis. Konstanz: UVK.

Gerbaudo, Paolo. 2018. Social media and populism: an elective affinity? Media, Culture \& Society 40(5):745-753.

Hameleers, Michael, und Cleas H. de Vreese. 2020. To whom are "the people" opposed? Conceptualizing and measuring citizens' populist attitudes as a multidimensional construct. Journal of Elections, Public Opinion and Parties 30(2):255-274.

Hameleers, Michael, Linda Bos, und Cleas H. de Vreese. 2017a. Shoot the messenger? The media's role in framing populist attributions of blame. Journalism 20(9):1-20.

Hameleers, Michael, Linda Bos, und Cleas H. de Vreese. 2017b. The appeal of media populism: the media preferences of citizens with populist attitudes. Mass Communciation \& Society 20(4):481-504.

Hawkins, Kirk A., Ryan E. Carlin, Levente Littvay, und Cristobal Rovira Kaltwasser. 2019. The ideational approach to populism: Concept, theory, and analysis. Routledge studies in extremism and democracy. London: Routledge.

Heiss, Raffael, und Jörg Matthes. 2019. Stuck in a nativist spiral: content, selection, and effects of rightwing populists' communication on Facebook. Political Communication 37(3):303-328.

Holsti, Ole. 1969. Content analysis for the social sciences and humanities. Reading: Addison-Wesley.

Hsueh, Mark, Kumar Yogeeswaran, und Sanna Malinen. 2015. "Leave your comment below": Can biased online comments influence our own prejudicial attitudes and behaviors? Human Communication Research 41(4):557-576.

Jagers, Jan, und Stefaan Walgrave. 2007. Populism as political communication style: An empirical study of political parties' discourse in Belgium. European Journal of Political Research 46(3):319-345.

van Kessel, Stijn, und Remco Castelein. 2016. "Shifting the blame. Populist politicians" use of Twitter as a tool of opposition. Journal of Contemporary European Research 12(2):594-614.

Krämer, Benjamin. 2018. Populism, media, and the form of society. Communication Theory 28(4):444-465.

Kriesi, Hanspeter. 2014. The populist challenge. West European Politics 37(2):361-378.

Laclau, Ernesto. 2005. On populist reason. London: Verso.

Lochoki, Timo. 2012. Immigration: Sprungbrett rechtspopulistischer Parteien. Aus Politik und Zeitgeschichte 62:30-36.

Mazzoleni, Gianpietro. 2008. Populism and the media. In Twenty-first century populism: The spectre of western European democracy, Hrsg. Daniele Albertazzi, Duncan McDonnell, 49-64. Basingstoke, New York: Palgrave Macmillan.

Mény, Yves, und Yves Surel. 2002. The constitutitive ambiguity of populism. In Democracies and the populist challenge, Hrsg. Yves Mény, Yves Surel, 1-21. Basingstoke: Palgrave Macmillan.

Meyer, Thomas. 2006. Populismus und Medien. In Populismus: Gefahr für die Demokratie oder nützliches Korrektiv?, Hrsg. Frank Decker, 81-96. Wiesbaden: Springer VS.

Moffitt, Benjamin. 2016. The global rise of populism: performance, political style, and representation. Palo Alto: Stanford University Press.

Mudde, Cas. 2004. The populist zeitgeist. Government and Opposition 39(4):542-563.

Mudde, Cas, und Cristobal Rovira Kaltwasser. 2017. Populism: A very short introduction. Oxford: Oxford University Press. 
Müller, Philipp, Christian Schemer, Martin Wettstein, Anne Schulz, Dominique S. Wirz, Sven Engesser, und Werner Wirth. 2017. The polarizing impact of news coverage on populist attitudes in the public: Evidence from a panel study in four European democracies. Journal of Communication 67(3):968-992.

Neuberger, Christoph. 2014. Konflikt, Konkurrenz und Kooperation: Interaktionsmodi in einer Theorie der dynamischen Netzwerköffentlichkeit. Medien \& Kommunikationswissenschaft 62(4):567-587.

Noelle-Neumann, Elisabeth. 1980. Die Schweigespirale. Öffentliche Meinung - unsere soziale Haut. Zürich, München: Piper.

Priester, Karin. 2011. Definitionen und Typologien des Populismus. Soziale Welt 62:185-198.

Priester, Karin. 2012. Wesensmerkmale des Populismus. Aus Politik und Zeitgeschichte 5(62):3-9.

Rauchenzauner, Elisabeth. 2008. Schlüsselereignisse in der Medienberichterstattung. Wiesbaden: Springer VS.

Reinemann, Carsten, Toril Aalberg, Frank Esser, Jesper Strömbäck, und Claes Vreese. 2017. Populist political communication: toward a model of its causes, forms, and effects. In Routledge research in communication studies. Populist political communication in europe, Hrsg. Toril Aalberg, Frank Esser, Carsten Reinemann, Jesper Strömbäck, und Claes H. de Vreese, 12-25. London: Routledge.

Rensmann, Lars. 2006. Populismus und Ideologie. In Populismus: Gefahr für die Demokratie oder nützliches Korrektiv, Hrsg. Frank Decker, 59-80. Wiesbaden: Springer VS.

Rössler, Patrick. 2010. Inhaltsanalyse, 2. Aufl., Opladen: UVK.

Sarcinelli, Ulrich. 2011. Politische Kommunikation in Deutschland. Medien und Politikvermittlung im demokratischen System. Wiesbaden: Springer VS.

Sarcinelli, Ulrich. 2013. Kommunikation und Partizipation in einer veränderten Legitimationsarchitektur. In Das Internet: Bereicherung oder Stressfaktor für die Demokratie?, Hrsg. Marianne Kneuer, 104-123. Baden-Baden: Nomos.

Sartori, Giovanni. 1976. Parties and party systems. A framework for analysis. Cambridge: ECPR Press.

Schindler, Johanna, Claudia Fortkord, Lone Posthumus, Magdalena Obermaier, Nayla Fawzi, und Carsten Reinemann. 2018. Woher kommt und wozu führt Medienfeindlichkeit? Zum Zusammenhang von populistischen Einstellungen, Medienfeindlichkeit, negativen Emotionen und Partizipation. Medien \& Kommunikationswissenschaft 66(3):283-301.

Schmuck, Desirée, und Michael Hameleers. 2019. Closer to the people: A comparative content analysis of populist communication on social networking sites in pre- and post-Election periods. Information, Communication \& Society. online first.

Schulz, Anne, und Patrick Rössler. 2012. Schweigespirale Online. Die Theorie der öffentlichen Meinung und das Internet. Baden-Baden: Nomos.

Spieß, Elina, 2020. Populistische Parteien- und Userkommunikation auf Facebook, unveröffentlichte Master-Arbeit, Münster.

Springer, Nina, Ines Engelmann, und Christian Pfaffinger. 2015. User comments: Motives and inhibitors to write and read. Information, Communication \& Society 18(7):798-815.

Staender, Anna, Nicole Ernst, und Desiree Steppat. 2019. Was steigert die Facebook-Resonanz? Eine Analyse der Likes, Shares und Comments im Schweizer Wahlkampf 2015. Studies in Communication and Media 8(2):236-271.

Stier, Sebastian, Arnim Bleier, Haiko Lietz, und Markus Strohmaier. 2018. Election campaigning on social media. Politicians, audiences, and the mediation of political communication on Facebook and twitter. Political Communication 35(1):50-74.

Stier, Sebastian, Lisa Posch, Arnim Bleier, und Markus Strohmaier. 2017. When populists become popular: comparing Facebook use by the right-wing movement Pegida and German political parties. Information, Communication \& Society 20(9):1365-1388.

Taggart, Paul. 2004. Populism and representative politics in contemporary Europe. Journal of Political Ideologies 9(3):269-288.

Waisbord, Silvio. 2018. Why populism is troubling for democratic communication. Communication, Culture and Critique 11(1):21-34.

Waisbord, Silvio, und Adriana Amado. 2017. Populist communication by digital means: presidential Twitter in Latin America. Information, Communication \& Society 20(9):1330-1346.

Wettstein, Martin, Frank Esser, Anne Schulz, Dominique S. Wirz, und Werner Wirth. 2018a. News media as gatekeepers, critics, and initiators of populist communication: how journalists in ten countries deal with the populist challenge. The International Journal of Press/Politic 23(4):476-495.

Wettstein, Martin, Frank Esser, Florian Büchel, Christian Schemer, Dominique S. Wirz, Anne Schulz, Nicole Ernst, Sven Engesser, Philipp Müller, und Werner Wirth. 2018b. What drives populist styles? 
Analyzing immigration and labor market news in 11 countries. Journalism \& Mass Communication Quarterly 96(2):516-536.

Winkler, Jürgen. 2013. Ursachen fremdenfeindlicher Einstellungen in Westeuropa. Aus Politik und Zeitgeschichte 26:33-38.

Wirth, Werner, Frank Esser, Martin Wettstein, Sven Engesser, Dominique S. Wirz, Anne Schulz, Nicole Ernst, Florin Büchel, Daniele Caramani, Luca Manucci, Marco Steenbergen, Bernhard Laurent, Edward Weber, Regula Hänggli, Caroline Dalmus, Christian Schemer, und Philipp Müller. 2016. The appeal of populist ideas, strategies and styles: A theoretical model and research design for analyzing populist political communication. NCCR Working Paper, Bd. 88. Zürich: National Centre of Competence in Research (NCCR).

Wirz, Dominique S. 2018. Persuasion through emotion? An experimental test of the emotion-eliciting nature of populist communication. International Journal of Communication 12:1114-1138.

Wirz, Dominique S., Martin Wettstein, Anne Schulz, Philipp Müller, Christian Schemer, Nicole Ernst, Frank Esser, und Werner Wirth. 2018. The effects of right-wing populist communication on emotions and Cognitions toward immigrants. The International Journal of Press/Politics 23(4):496-516.

Wolling, Jens. 2005. Normalzeit vs. Spezialzeit. Besondere Ereignisse als Problem der Stichprobenziehung bei Inhaltsanalysen von Medienangeboten. In Auswahlverfahren in der Kommunikationswissenschaft, Hrsg. Volker Gehrau, 138-157. Köln: Halem.

Ziegele, Marc, Christina Koehler, und Mathias Weber. 2018. Socially destructive? Effects of negative and hateful user comments on readers' donation behavior toward refugees and homeless persons. Journal of Broadcasting \& Electronic Media 62(4):636-653.

Ziegele, Marc, Teresa Naab, und Pablo Jost. 2020. Lonely together? Identifying the determinants of collective corrective action against uncivil comments. New Media \& Society 22(5):731-751. 